\title{
Adiabatic invariants and some statistical properties of the time-dependent linear and nonlinear oscillators
}

\author{
M. Robnik
}

\author{
CAMTP - Center for Applied Mathematics and Theoretical Physics, University of \\ Maribor, Maribor, Slovenia, European Union,e-mail: Robnik@uni-mb.si
}

We consider 1D time dependent Hamiltonian systems and their statistical properties, namely the time evolution of microcanonical distributions, whose properties are very closely related to the existence and preservation of the adiabatic invariants. We review the elements of the recent developments by Robnik and Romanovski (2006-2008) for the entirely general 1D time dependent linear oscillator and try to generalize the results to the 1D nonlinear Hamilton oscillators, in particular the power-law potentials like e.g. quartic oscillator. Furthermore we consider the limit opposite to the adiabatic limit, namely parametrically kicked 1D Hamiltonian systems. Even for the linear oscillator interesting properties are revealed: an initial kick disperses the microcanonical distribution to a spread one, but an anti-kick at an appropriate moment of time can annihilate it, kicking it back to the microcanonical distribution. The case of periodic parametric kicking is also interesting. Finally we propose that in the parametric kicking of a general 1D Hamilton system the average value of the adiabatic invariant always increases, which we prove for the power-law potentials. We find that the approximation of kicking is good for quite long times of the parameter variation, up to the order of not much less than one period of the oscillator. We also look at the behaviour of the quartic oscillator for the case of the kick and anti-kick, and also the periodic kicking.

\author{
References: \\ Papamikos G and Robnik M 2012 J.Phys.A: Math.Theor. 44315102 \\ Robnik M and Romanovski V G 2006 J.Phys.A: Math.Gen. 39 L35-L41 \\ Robnik M and Romanovski V G 2006 Open Sys. and Inf. Dynamics 13 197-222 \\ Robnik M, Romanovski V G and Stockmann H.-J. 2006 J.Phys.A:Math.Gen. 39 L551- \\ L554 \\ Kuzmin A V and Robnik M 2007 Rep.Math.Phys. 60 69-84 \\ Robnik M V and Romanovski V G 2008 "Let's Face Chaos through Nonlinear \\ Dynamics", \\ Proceedings of the 7th International summer school/conference, Maribor-Slovenia, \\ 2008, in AIP Conf. Proc. 1076, Eds. M.Robnik and V.G. Romanovski. \\ Robnik M and Romanovski V G 2000 J.Phys.A: Math.Theor. 335093
}

